Marquette University

e-Publications@Marquette

2018

\title{
On Extended Quadratic Hazard Rate Distribution: Development, Properties, Characterizations and Applications
}

\author{
Fiaz Ahmad Bhatti \\ National College of Business Administration and Economic \\ Gholamhossein G. Hamedani \\ Marquette University, gholamhoss.hamedani@marquette.edu \\ Wenhui Sheng \\ Marquette University \\ Munir Ahmad \\ National College of Business Administration and Economics, Lahore
}

Follow this and additional works at: https://epublications.marquette.edu/math_fac

\section{Recommended Citation}

Bhatti, Fiaz Ahmad; Hamedani, Gholamhossein G.; Sheng, Wenhui; and Ahmad, Munir, "On Extended Quadratic Hazard Rate Distribution: Development, Properties, Characterizations and Applications" (2018). Mathematical and Statistical Science Faculty Research and Publications. 10.

https://epublications.marquette.edu/math_fac/10 


\title{
Research Article
}

Fiaz Ahmad Bhatti*, G. G. Hamedani, Wenhui Sheng and Munir Ahmad

\section{On Extended Quadratic Hazard Rate Distribution: Development, Properties, Characterizations and Applications}

https://doi.org/10.1515/eqc-2018-0002

Received January 13, 2018; revised March 5, 2018; accepted March 8, 2018

\begin{abstract}
In this paper, we propose a flexible extended quadratic hazard rate (EQHR) distribution with increasing, decreasing, bathtub and upside-down bathtub hazard rate function. The EQHR density is arc, rightskewed and symmetrical shaped. This distribution is also obtained from compounding mixture distributions. Stochastic orderings, descriptive measures on the basis of quantiles, order statistics and reliability measures are theoretically established. Characterizations of the EQHR distribution are studied via different techniques. Parameters of the EQHR distribution are estimated using the maximum likelihood method. Goodness of fit of this distribution through different methods is studied.
\end{abstract}

Keywords: Stochastic Ordering, Reliability, Characterizations, Compounding, Maximum Likelihood Estimation

MSC 2010: 60E05, 62E10, 62E15,62H12, 68U05, 68U20

\section{Introduction}

During recent decades, many continuous univariate distributions have been developed but various data sets from reliability, engineering, environmental, financial, biomedical sciences, among other areas, do not follow these distributions. Therefore, modified, extended and generalized distributions and their applications to problems in these areas are required.

The modified, extended and generalized distributions are obtained by the introduction of some transformation or additional one or more parameters to the baseline distribution. These new developed distributions provide better fit to data than the baseline model.

The main purpose of this article is to develop a more flexible distribution called EQHR distribution. The EQHR distribution has increasing, decreasing, bathtub and upside-down bathtub hazard rate function. The flexible nature of the hazard rate function of the EQHR distribution helps to serve as the best alternative model to other current models for modeling real data in reliability, survival analysis, economics, life testing and other areas of research. The EQHR distribution provides better fits than the baseline model.

Our interest is to study the EQHR distribution in terms of its mathematical properties, applications and comparison to sub-models.

*Corresponding author: Fiaz Ahmad Bhatti, National College of Business Administration and Economics, Lahore, Pakistan, e-mail: fiazahmad72@gmail.com

G. G. Hamedani, Wenhui Sheng, Marquette University, Milwaukee, WI 53201-1881, USA,

e-mail: g.hamedani@mu.edu,wenhui.sheng@marquette.edu

Munir Ahmad, National College of Business Administration and Economics, Lahore, Pakistan,

e-mail: munirahmaddr@yahoo.co.uk 
The article is composed of the following sections. In Section 2, the EQHR distribution is introduced. In Section 3, the EQHR distribution is studied in terms of the basic structural properties, sub-models, plots, stochastic ordering and descriptive measures on the basis of quantiles and moments. In Section 4, probability density functions for different order statistics are developed. In Section 5, reliability measures are studied. In Section 6, characterizations of the EQHR distribution are presented via (i) conditional expectation; (ii) truncated moment; (iii) hazard function; (iv) Mills ratio; (v) certain functions of the random variable; (vi) first-order statistic; and (vii) conditional expectation of record values. In Section 7, the EQHR distribution is derived through compounding (i) generalized quadratic hazard rate (GQHR) and gamma distributions and (ii) GQHR and sized biased moment Erlang distributions. In Section 8, simulation study and MLE (maximum likelihood estimation) is carried out. In Section 9, the potentiality of the EQHR distribution is demonstrated by its applications to real data sets; parameters of EQHR are estimated using the maximum likelihood method. Goodness of fit of the probability distribution through different methods is studied. Finally, in Section 10, we provide some concluding remarks.

\section{EQHR Distribution}

The cumulative distribution function (cdf) for random variable $Y$ having Lomax distribution is given by

$$
F_{L}(y)=1-(1+y)^{-\kappa}, \quad y \geq 0, \kappa>0 .
$$

The probability density function (pdf) of the Lomax distribution for random variable $Y$ is

$$
f_{L}(y)=\kappa(1+y)^{-(\kappa+1)}, \quad y>0, \kappa>0 .
$$

Bain [5] has developed a quadratic hazard rate (QHR) distribution using the following quadratic function:

$$
Q(x \mid \alpha, \beta, \gamma)=\alpha+\beta x+\gamma x^{2}, \quad x>0 .
$$

The cdf of a random variable $X$ following the QHR distribution with parameters $\alpha, \beta$ and $\gamma$ is given by

$$
F_{\mathrm{QHR}}(x)=1-e^{-\left(\alpha x+\frac{\beta}{2} x^{2}+\frac{\gamma}{3} x^{3}\right)}, \quad \alpha>0, y>0, \beta>-2 \sqrt{\alpha y}, \quad x \geq 0 .
$$

The corresponding pdf of $X$ is

$$
f_{\mathrm{QHR}}(x)=\left(\alpha+\beta x+\gamma x^{2}\right) e^{-\left(\alpha x+\frac{\beta}{2} x^{2}+\frac{\gamma}{3} x^{3}\right)}, \quad x>0 .
$$

Sarhan [21] introduced the generalized quadratic hazard rate distribution. Elbatal and Butt [9] studied the generalization of the quadratic hazard rate distribution and its properties. Merovci and Elbatal [15] studied the beta quadratic hazard rate distribution and its properties. Okasha et al. [19] presented the quadratic hazard rate geometric distribution and its properties. El-Damcese and Ramadan [8] analyzed reliability results about the generalized quadratic hazard rate truncated Poisson Maximum distribution. Roozegar and Nadarajah [20] introduced the quadratic hazard rate power series distribution and studied its properties. Kayid, Elbatal and Merovci [13] presented a generalized family of the quadratic hazard rate distribution and studied its applications. Mustafa [17] derived a bivariate distribution with generalized quadratic hazard rate marginals. Al-Hossain and Dar [2] presented moment of order statistics for the quadratic hazard rate function. Bhatti and Ahmad [7] characterized the quadratic hazard rate-geometric distribution via different techniques.

The EQHR distribution is proposed by inducting the generator of QHR distribution in the cdf (2.1) to get the form

$$
F(x)=1-[A(x \mid \alpha, \beta, \gamma)]^{-\kappa}, \quad x \geq 0,
$$

where

$$
A(x \mid \alpha, \beta, \gamma)=1+\alpha x+\beta \frac{x^{2}}{2}+\gamma \frac{x^{3}}{3}
$$

and $\alpha, \beta, \kappa$ and $\gamma$ are parameters. The EQHR density is

$$
f(x, \alpha, \beta, \gamma, \kappa)=\kappa Q(x \mid \alpha, \beta, \gamma)[A(x \mid \alpha, \beta, \gamma)]^{-(\kappa+1)}, \quad x>0 .
$$




\section{Structural Properties of EQHR Distribution}

The survival, hazard, cumulative hazard and reverse hazard functions of a random variable $X$ with EQHR distribution are given, respectively, by

$$
\begin{aligned}
S(x) & =[A(x \mid \alpha, \beta, \gamma)]^{-\kappa}, \quad x \geq 0, \\
h(x) & =\kappa Q(x \mid \alpha, \beta, \gamma)[A(x \mid \alpha, \beta, \gamma)]^{-1}, \quad x>0, \\
H(x) & =\kappa \ln [A(x \mid \alpha, \beta, \gamma)], \quad x>0, \\
r(x) & =\frac{f(x)}{F(x)}=\frac{\kappa Q(x \mid \alpha, \beta, \gamma)[A(x \mid \alpha, \beta, \gamma)]^{-(\kappa+1)}}{1-[A(x \mid \alpha, \beta, \gamma)]^{-\kappa}}, \quad x>0 .
\end{aligned}
$$

The Mills ratio of the EQHR distribution is

$$
m(x)=\frac{1-F(x)}{f(x)}=\kappa^{-1}[Q(x \mid \alpha, \beta, \gamma)]^{-1}[A(x \mid \alpha, \beta, \gamma)] .
$$

The elasticity $e(x)=\frac{d \ln F(x)}{d \ln x}=x r(x)$ of the EQHR distribution is given by

$$
e(x)=\frac{\kappa Q(x \mid \alpha, \beta, \gamma)[A(x \mid \alpha, \beta, \gamma)]^{-(\kappa+1)}}{1-[A(x \mid \alpha, \beta, \gamma)]^{-\kappa}} .
$$

The elasticity of the EQHR distribution shows the behavior of the accumulation of probability in the domain of the random variable.

The mode of the EQHR distribution with pdf (2.3) is obtained by solving $\frac{d}{d x}[\ln f(x)]=0$, i.e.,

$$
\left\{\frac{(\beta+2 \gamma x)}{Q(x \mid \alpha, \beta, \gamma)}-\frac{(\kappa+1) Q(x \mid \alpha, \beta, \gamma)}{A(x \mid \alpha, \beta, \gamma)}\right\}=0 .
$$

\subsection{Sub-Models of EQHR Distribution}

The EQHR distribution has wide applications in life testing, survival analysis, and reliability theory. The submodels of the EQHR are shown in Table 1.

\begin{tabular}{cccccl}
\hline & $\boldsymbol{\alpha}$ & $\boldsymbol{\beta}$ & $\boldsymbol{\gamma}$ & $\boldsymbol{\kappa}$ & Name of distribution \\
\hline 1 & $\alpha$ & 0 & 0 & $\kappa$ & Extended exponential \\
2 & 0 & $\beta$ & 0 & $\kappa$ & Extended Rayleigh \\
3 & 0 & 0 & $\gamma$ & $\kappa$ & Extended Weibull \\
4 & $\alpha$ & $\beta$ & $\gamma$ & $\kappa$ & EQHR distribution \\
5 & $\alpha$ & $\beta$ & 0 & $\kappa$ & Linear failure rate \\
\hline
\end{tabular}

Table 1: Sub-models of the EQHR.

\subsection{Shapes of the EQHR Density and Hazard Rate Function}

The shapes of the EQHR density are arc, positively skewed and symmetrical, see Figure 1 . The EQHR distribution has increasing, decreasing, bathtub and upside-down bathtub hazard rate function, see Figure 2 .

\subsection{Stochastic Ordering}

Stochastic orderings are used for comparative behavior. 


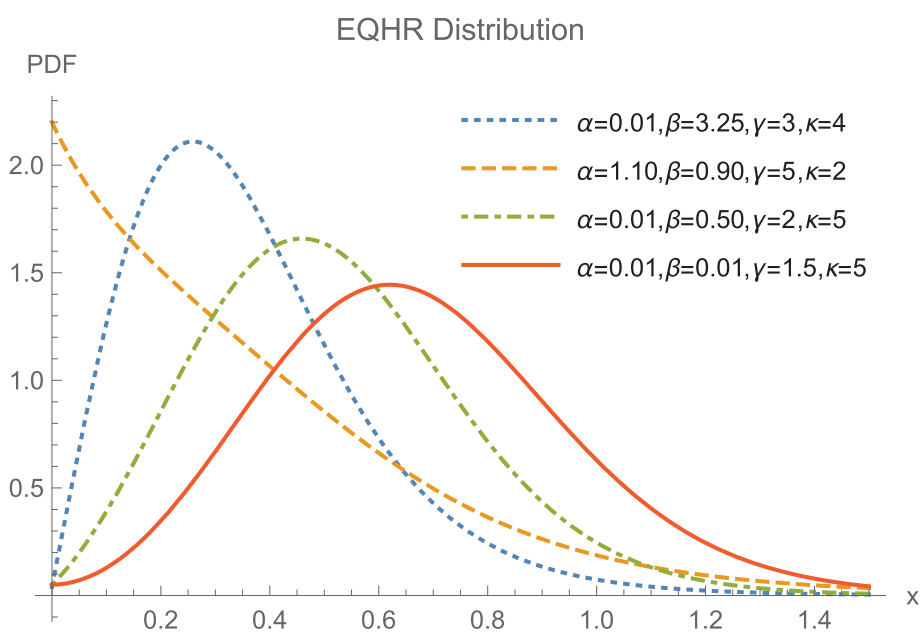

Figure 1: Plot of pdf of EQHR distribution.

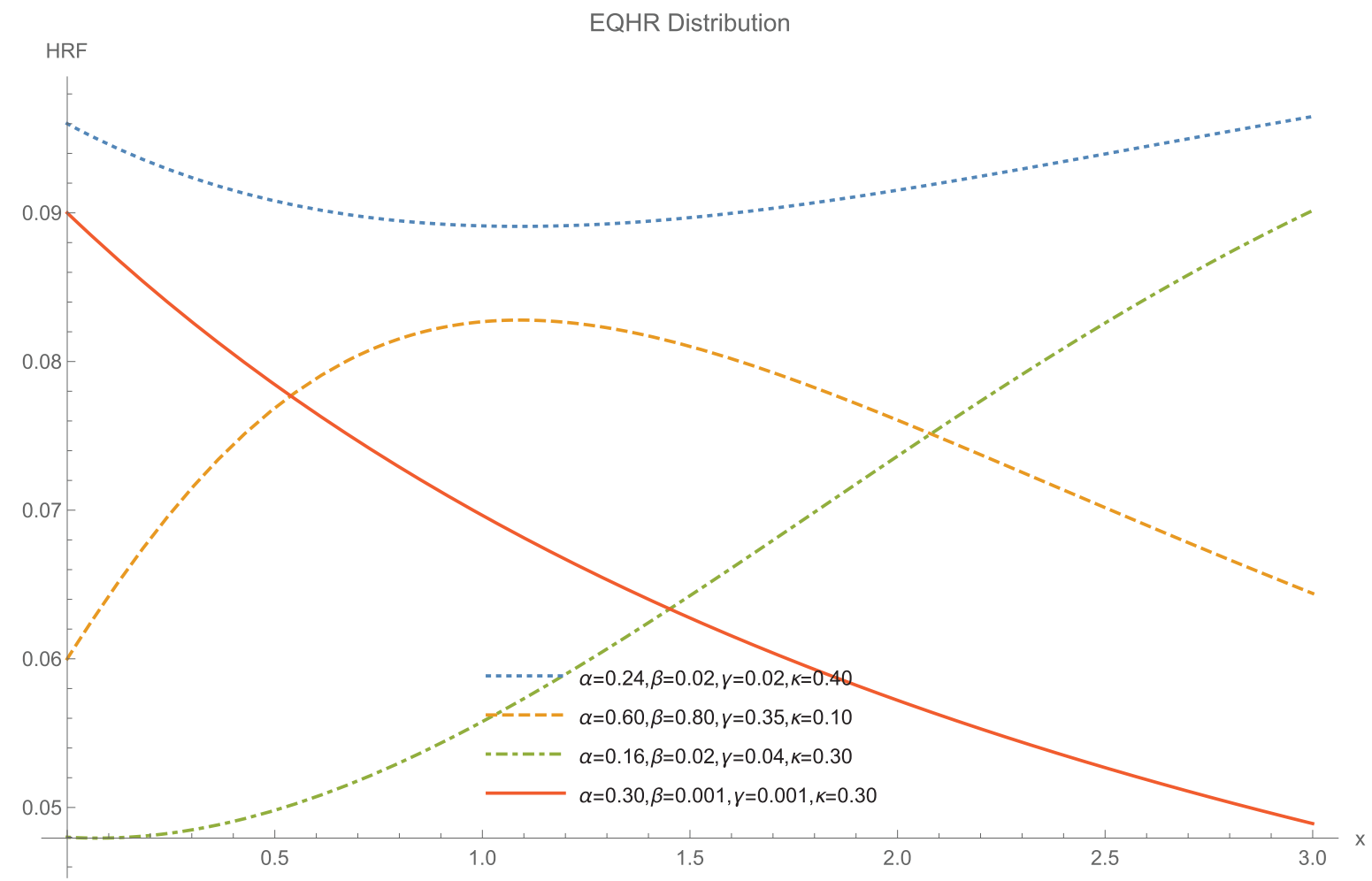

Figure 2: Plots of hrf of EQHR distribution.

Definition 3.1. A random variable $X$ is said to be smaller than a random variable $Y$ in

(i) stochastic order, $X \leq_{\mathrm{st}} Y$, if $F_{X}(x) \geq F_{Y}(x)$ for all $x$,

(ii) likelihood ratio order, $X \leq_{\operatorname{lr}} Y$, if $f_{X}(x) / f_{Y}(x)$ decreases in $x$,

(iii) hazard rate order, $X \leq{ }_{\mathrm{hr}} Y$, if $h_{X}(x) \geq h_{Y}(x)$ for all $x$,

(iv) mean residual life order, $X \leq_{\operatorname{mrl}} Y$, if $m_{X}(x) \geq m_{Y}(x)$ for all $x$.

Theorem 3.2. Let $X$ and $Y$ be random variables with EQHR distributions. For $\alpha_{1}=\alpha_{2}, \beta_{1}=\beta_{2}, \gamma_{1}=\gamma_{2}$ and $\kappa_{1}>\kappa_{2}$, the random variable $X$ is said to be smaller than the random variable $Y$ in likelihood ratio order, $X \leq_{1 \mathrm{r}} Y$, if $\frac{d}{d x}\left[\ln \left(f_{X}(x) / f_{Y}(x)\right)\right]<0$. Then $X \leq_{\mathrm{lr}} Y \Rightarrow X \leq_{\mathrm{hr}} Y \Rightarrow X \leq_{\mathrm{mrl}} Y \Rightarrow X \leq_{\mathrm{st}} Y$. 
Proof. We have

$$
\begin{aligned}
f_{X}(x) & =\kappa_{1} Q\left(x \mid \alpha_{1}, \beta_{1}, \gamma_{1}\right)\left[A\left(x \mid \alpha_{1}, \beta_{1}, \gamma_{1}\right)\right]^{-\kappa_{1}-1}, \quad x>0, \\
f_{Y}(x) & =\kappa_{2} Q\left(x \mid \alpha_{2}, \beta_{2}, \gamma_{2}\right)\left[A\left(x \mid \alpha_{2}, \beta_{2}, \gamma_{2}\right)\right]^{-\kappa_{2}-1}, \quad x>0, \\
\frac{f_{X}(x)}{f_{Y}(x)} & =\frac{\kappa_{1} Q\left(x \mid \alpha_{1}, \beta_{1}, \gamma_{1}\right)\left[A\left(x \mid \alpha_{1}, \beta_{1}, \gamma_{1}\right)\right]^{-\kappa_{1}-1}}{\kappa_{2} Q\left(x \mid \alpha_{2}, \beta_{2}, \gamma_{2}\right)\left[A\left(x \mid \alpha_{2}, \beta_{2}, \gamma_{2}\right)\right]^{-\kappa_{2}-1}}, \\
\frac{d}{d x}\left[\ln \frac{f_{X}(x)}{f_{Y}(x)}\right] & =\frac{\beta_{1}+2 \gamma_{1} x}{Q\left(x \mid \alpha_{1}, \beta_{1}, \gamma_{1}\right)}-\frac{\left(\beta_{2}+2 \gamma_{2} x\right)}{Q\left(x \mid \alpha_{2}, \beta_{2}, \gamma_{2}\right)}-\left(\kappa_{1}+1\right) \frac{Q\left(x \mid \alpha_{1}, \beta_{1}, \gamma_{1}\right)}{A\left(x \mid \alpha_{1}, \beta_{1}, \gamma_{1}\right)}+\left(\kappa_{2}+1\right) \frac{Q\left(x \mid \alpha_{2}, \beta_{2}, \gamma_{2}\right)}{A\left(x \mid \alpha_{2}, \beta_{2}, \gamma_{2}\right)} .
\end{aligned}
$$

Then, for $\alpha_{1}=\alpha_{2}, \beta_{1}=\beta_{2}, \gamma_{1}=\gamma_{2}, \kappa_{1}>\kappa_{2}$,

$$
\frac{d}{d x}\left[\ln \frac{f_{X}(x)}{f_{Y}(x)}\right]<0 .
$$

It means that the random variable $X$ is smaller than the random variable $Y$ in likelihood ratio order, $X \leq_{\operatorname{lr}} Y$, because $\frac{d}{d x}\left[\ln \left(f_{X}(x) / f_{Y}(x)\right)\right]<0$. Therefore the EQHR distribution is ordered strongly with respect to stochastic ordering.

Theorem 3.3. Let $X$ and $Y$ be random variables having EQHR distributions. For $\alpha_{1}=\alpha_{2}, \beta_{1}=\beta_{2}, \gamma_{1}=\gamma_{2}$ and $\kappa_{1}>\kappa_{2}$, the random variable $X$ is said to be smaller than the random variable $Y$ in likelihood ratio order, $X \leq_{1 \mathrm{r}} Y$, if $\frac{d}{d x}\left[\ln \left(\bar{F}_{X}(x) / \bar{F}_{Y}(x)\right)\right]<0$. Then $X \leq_{\mathrm{lr}} Y \Rightarrow X \leq_{\mathrm{hr}} Y \Rightarrow X \leq_{\mathrm{mrl}} Y \Rightarrow X \leq_{\mathrm{st}} Y$.

Proof. We have

$$
\bar{F}_{X}(x)=\left[A\left(x \mid \alpha_{1}, \beta_{1}, \gamma_{1}\right)\right]^{-\kappa_{1}} \quad \text { and } \quad \bar{F}_{Y}(x)=\left[A\left(x \mid \alpha_{2}, \beta_{2}, \gamma_{2}\right)\right]^{-\kappa_{2}},
$$

and

$$
\frac{\bar{F}_{X}(x)}{\bar{F}_{Y}(x)}=\frac{\left[A\left(x \mid \alpha_{1}, \beta_{1}, \gamma_{1}\right)\right]^{-\kappa_{1}}}{\left[A\left(x \mid \alpha_{2}, \beta_{2}, \gamma_{2}\right)\right]^{-\kappa_{2}}}, \quad \frac{d}{d x}\left[\ln \frac{\bar{F}_{X}(x)}{\bar{F}_{Y}(x)}\right]=-\frac{\kappa_{1}\left(\beta_{1}+2 \gamma_{1} x\right)}{Q\left(x \mid \alpha_{1}, \beta_{1}, \gamma_{1}\right)}+\frac{\kappa_{2}\left(\beta_{2}+2 \gamma_{2} x\right)}{Q\left(x \mid \alpha_{2}, \beta_{2}, \gamma_{2}\right)} .
$$

Then, for $\alpha_{1}=\alpha_{2}, \beta_{1}=\beta_{2}, \gamma_{1}=\gamma_{2}, \kappa_{1}>\kappa_{2}$,

$$
\frac{d}{d x}\left[\ln \frac{\bar{F}_{X}(x)}{\bar{F}_{Y}(x)}\right]<0 .
$$

It means that the random variable $X$ is smaller than the random variable $Y$ in likelihood ratio order, $X \leq_{\operatorname{lr}} Y$, because $\frac{d}{d x}\left[\ln \left(\bar{F}_{X}(x) / \bar{F}_{Y}(x)\right)\right]<0$. Therefore the EQHR distribution is ordered strongly with respect to stochastic ordering.

\subsection{Descriptive Measures Based on Quantiles}

The quantile function of the EQHR distribution is the solution of $A(x \mid \alpha, \beta, \gamma)=[1-q]^{-1 / \kappa}$. The median of the EQHR distribution is the solution of $A(x \mid \alpha, \beta, \gamma)=2^{1 / \kappa}$ and the random number generator $X$ for the EQHR distribution is the solution of $A(X \mid \alpha, \beta, \gamma)=[1-Z]^{-1 / \kappa}$, where the random variable $Z$ has uniform distribution on $(0,1)$.

Some measures based on quartiles for location, dispersion, skewness and kurtosis, respectively, for the EQHR distribution are:

- median

$$
M=Q_{1 / 2},
$$

- quartile deviation

$$
\text { Q.D. }=\frac{Q_{3 / 4}-Q_{1 / 4}}{2},
$$

- Bowley's skewness measure

$$
S_{q}=\frac{Q_{3 / 4}-2 Q_{1 / 2}+Q_{1 / 4}}{Q_{3 / 4}-Q_{1 / 4}},
$$


- Moors kurtosis measure based on octiles

$$
K=\frac{Q_{7 / 8}-Q_{5 / 8}+Q_{3 / 8}-Q_{1 / 8}}{Q_{6 / 8}-Q_{2 / 8}} .
$$

Quantile based measures exist even for distributions that are without moments. Quantile based measures are less sensitive to outliers.

\subsection{Moments}

The $r$ th raw moments for the EQHR distribution can be obtained from

$$
\begin{aligned}
& E\left(X^{r}\right)=\int_{0}^{\infty} x^{r} d F(x), \\
& E\left(X^{r}\right)=\int_{0}^{\infty} r x^{r-1}[A(x \mid \alpha, \beta, \gamma)]^{-\kappa} d x \quad \text { for } \gamma<3 \kappa .
\end{aligned}
$$

The moment generating function is as follows:

$$
M_{X}(t)=E\left(e^{t X}\right)=\int_{0}^{\infty} e^{t x} \kappa Q(x \mid \alpha, \beta, \gamma)[A(x \mid \alpha, \beta, \gamma)]^{-\kappa-1} d x .
$$

The $r$ th incomplete moments for the EQHR distribution can be obtained from

$$
I(y ; r)=E\left(X^{r} \mid X \leq y\right)=(F(y))^{-1} \int_{0}^{y} x^{r} \kappa Q(x \mid \alpha, \beta, \gamma)[A(x \mid \alpha, \beta, \gamma)]^{-\kappa-1} d x,
$$

where $F(x)$ is the cdf of $X$.

\section{Order Statistics}

The order statistics mostly appear in the problems of estimation and testing. The application of extreme values is very common in reliability, meteorology, econometrics and various areas of research.

The $\operatorname{pdf} f_{X_{j: n}}(x)$ of $j$ th order statistic $X_{j: n}$, from a cdf $F$ with $\operatorname{pdf} f$, is

$$
f_{X_{j: n}}(x)=\frac{1}{B(j, n-j+1)}[F(x)]^{j-1}[1-F(x)]^{n-j} f(x) .
$$

The $\operatorname{pdf} f_{X_{j: n}}(x)$ of $j$ th order statistic $X_{j: n}$ for the EQHR distribution is given by

$$
f_{X_{j: n}}(x)=\frac{1}{B(j, n-j+1)} \sum_{i=0}^{j-1}(-1)^{i}\left(\begin{array}{c}
j-1 \\
i
\end{array}\right) \kappa Q(x \mid \alpha, \beta, \gamma)[A(x \mid \alpha, \beta, \gamma)]^{-(k n+k i-k j+k+1)} .
$$

The $\operatorname{pdf} f_{X_{n: n}}(x)$ of $n$th order statistic $X_{n: n}$, from a cdf $F$ with $\operatorname{pdf} f$, is

$$
f_{X_{n: n}}(x)=n[F(x)]^{n-1} f(x) .
$$

The $\operatorname{pdf} f_{X_{n: n}}(x)$ of $n$th order statistic $X_{n: n}$ for the EQHR distribution is given by

$$
f_{X_{n: n}}(x)=\kappa n \sum_{i=0}^{n-1}(-1)^{i}\left(\begin{array}{c}
n-1 \\
i
\end{array}\right) Q(x \mid \alpha, \beta, \gamma)[A(x \mid \alpha, \beta, \gamma)]^{-(\kappa+\kappa i+1)} .
$$

The $\operatorname{pdf} f_{X_{1: n}}(x)$ of first-order statistic $X_{1: n}$, from a $\operatorname{cdf} F$ with $\operatorname{pdf} f$, is

$$
f_{X_{1: n}}(x)=n[1-F(x)]^{n-1} f(x) .
$$

The $\operatorname{pdf} f_{X_{1: n}}(x)$ of first-order statistic $X_{1: n}$ for the EQHR distribution is given by

$$
f_{X_{1: n}}(x)=n \kappa Q(x \mid \alpha, \beta, \gamma)[A(x \mid \alpha, \beta, \gamma)]^{-(\kappa n+1)} .
$$




\section{Reliability Measures}

In this section, reliability measures are studied.

\subsection{Stress-Strength Reliability for EQHR Distribution}

If $X_{1} \sim \operatorname{EQHR}\left(\alpha, \beta, \gamma, \kappa_{1}\right)$ and $X_{2} \sim \operatorname{EQHR}\left(\alpha, \beta, \gamma, \kappa_{2}\right)$ such that $X_{1}$ represents "strength" and $X_{2}$ represents "stress" and $X_{1}, X_{2}$ follow a joint pdf $f\left(x_{1}, x_{2}\right)$, then $R=\operatorname{Pr}\left(X_{2}<X_{1}\right)$ is a reliability parameter. Thus $R=\operatorname{Pr}\left(X_{2}<X_{1}\right)=\int_{0}^{\infty} f_{X_{1}}(x) F_{X_{2}}(x) d x$ is the characteristic of the distribution of $X_{1}$ and $X_{2}$. The reliability of the component is computed using pdf (2.3) and cdf (2.2) of the EQHR distribution as

$$
\begin{aligned}
& R=\int_{0}^{\infty} f_{x_{1}}(x) F_{X_{2}}(x) d x, \\
& R=\int_{0}^{\infty} \kappa_{1} Q(x \mid \alpha, \beta, \gamma)[A(x \mid \alpha, \beta, \gamma)]^{-\kappa_{1}-1}\left[1-[A(x \mid \alpha, \beta, \gamma)]^{-\kappa_{2}}\right] d x, \\
& R=1-\int_{0}^{\infty} \kappa_{1} Q(x \mid \alpha, \beta, \gamma)[A(x \mid \alpha, \beta, \gamma)]^{-\kappa_{1}-\kappa_{2}-1} d x, \\
& R=\frac{\kappa_{2}}{\left(\kappa_{1}+\kappa_{2}\right)} .
\end{aligned}
$$

Therefore, $R$ is independent of $\alpha, \beta$ and $\gamma$; and $R=0.5$ for $\kappa_{1}=\kappa_{2}$. It means that $X_{1}$ and $X_{2}$ are independently identically distributed (i.i.d.) and $\operatorname{Pr}\left(X_{1}>X_{2}\right)=\operatorname{Pr}\left(X_{1}<X_{2}\right)$.

\subsection{Estimation of Multicomponent Stress-Strength System Reliability for EQHR Distribution}

Suppose a machine has at least $s$ components working out of $m$ components. The strengths of all components of the system are $X_{1}, X_{2}, \ldots, X_{k}$ and stress $Y$ is applied on the system. The strengths $X_{1}, X_{2}, \ldots, X_{k}$ are both i.i.d. and independent of stress $Y$. The cdf of $Y$ is $G$, and the cdf of $X$ is $F$. The reliability of a machine is the probability that the machine functions properly, i.e.

$$
R_{s, k}=P(\text { strengths }>\text { stress })=P\left[\text { at least } s \text { of }\left(X_{1}, X_{2}, \ldots, X_{k}\right) \text { exceed } Y\right] .
$$

Let $X \sim \operatorname{EQHR}\left(\alpha, \beta, \gamma, \kappa_{1}\right), Y \sim \operatorname{EQHR}\left(\alpha, \beta, \gamma, \kappa_{2}\right)$ with unknown shape parameters $\kappa_{1}$ and $\kappa_{2}$ and common scale parameters $\alpha, \beta, \gamma$. The reliability in multicomponent stress-strength for the EQHR distribution is given by

$$
R_{s, m}=\sum_{l=s}^{m}\left(\begin{array}{c}
m \\
l
\end{array}\right) \int_{0}^{\infty}[1-F(y)]^{l}[F(y)]^{m-l} d G(y)
$$

(see Bhattacharyya and Johnson [6]) and

$$
R_{s, m}=\sum_{\ell=s}^{m}\left(\begin{array}{c}
m \\
\ell
\end{array}\right) \int_{0}^{\infty}\left([A(y \mid \alpha, \beta, \gamma)]^{-\kappa_{1}}\right)^{\ell}\left(1-[A(y \mid \alpha, \beta, \gamma)]^{-\kappa_{1}}\right)^{(m-\ell)} \kappa_{2} Q(y \mid \alpha, \beta, \gamma)[A(y \mid \alpha, \beta, \gamma)]^{-\kappa_{2}-1} d y .
$$

Let $t=[A(y \mid \alpha, \beta, \gamma)]^{-\kappa_{2}}$. Then we arrive at

$$
R_{s, m}=\sum_{\ell=S}^{m}\left(\begin{array}{c}
m \\
\ell
\end{array}\right) \int_{0}^{1}\left(t^{v}\right)^{\ell}\left(1-t^{v}\right)^{(m-\ell)} d t
$$


where

$$
v=\frac{\kappa_{1}}{\kappa_{2}}
$$

Let

$$
t^{v}=z, \quad t=z^{\frac{1}{v}}, \quad \frac{1}{v} z^{\frac{1}{v}-1} d z=d t .
$$

Then we obtain

$$
\begin{aligned}
& R_{S, m}=\sum_{\ell=S}^{m}\left(\begin{array}{c}
m \\
\ell
\end{array}\right) \int_{0}^{1}(z)^{\ell}(1-z)^{(m-\ell)} \frac{1}{v} z^{\frac{1}{v}-1} d z, \\
& R_{S, m}=\frac{1}{v} \sum_{\ell=S}^{m}\left(\begin{array}{c}
m \\
\ell
\end{array}\right) B\left(\ell+\frac{1}{v}, m-\ell+1\right)=\frac{1}{v} \sum_{\ell=S}^{m} \frac{m !}{(m-\ell) !}\left(\prod_{j=0}^{\ell}(m+v-j)\right)^{-1} .
\end{aligned}
$$

The probability $R_{s, m}$ in (5.1) is called multicomponent stress-strength model reliability.

\section{Characterization}

In this section, the EQHR distribution is characterized via (i) conditional expectation; (ii) truncated moment; (iii) hazard function; (iv) Mills ratio; (v) certain functions of the random variable; (vi) first-order statistic and (vii) conditional expectation of record values.

We present our characterizations in seven subsections.

\subsection{Characterization Based on Conditional Expectation}

Here is our first characterization of the EQHR distribution.

Proposition 6.1. Let $X: \Omega \rightarrow(0, \infty)$ be a continuous random variable with cdf $F(x)(0<F(x)<1$ for $x>0)$, then for $\kappa>1, X$ has $c d f(2.2)$ if and only if

$$
E[(A(X \mid \alpha, \beta, \gamma)-1) \mid X>t]=\left\{\frac{\kappa}{\kappa-1} A(t \mid \alpha, \beta, \gamma)-1\right\} \quad \text { for } \kappa>1,
$$

holds.

Proof. If $X$ has cdf (2.2), then

$$
\begin{aligned}
E[(A(X \mid \alpha, \beta, \gamma)-1) \mid X>t] & =(1-F(t))^{-1} \int_{t}^{\infty}(A(x \mid \alpha, \beta, \gamma)-1) f(x) d x \\
& =[A(t \mid \alpha, \beta, \gamma)]^{\kappa} \int_{t}^{\infty}(A(x \mid \alpha, \beta, \gamma)-1) \times \kappa Q(x \mid \alpha, \beta, \gamma)[A(x \mid \alpha, \beta, \gamma)]^{-(\kappa+1)} d x .
\end{aligned}
$$

Upon integration by parts and simplification, we arrive at

$$
E[(A(X \mid \alpha, \beta, \gamma)-1) \mid X>t]=\left\{\frac{\kappa}{\kappa-1} A(t \mid \alpha, \beta, \gamma)-1\right\} \quad \text { for } \kappa>1,
$$

Conversely, if (6.1) holds, then

$$
\begin{aligned}
(1-F(t))^{-1} \int_{t}^{\infty}(A(x \mid \alpha, \beta, \gamma)-1) f(x) d x & =\left\{\frac{\kappa}{\kappa-1} A(t \mid \alpha, \beta, \gamma)-1\right\}, \\
\int_{t}^{\infty}(A(x)-1) f(x) d x & =(1-F(t))\left\{\frac{\kappa}{\kappa-1} A(t)-1\right\} .
\end{aligned}
$$


Differentiating both sides of the above equation with respect to $t$, we obtain

$$
-(A(t \mid \alpha, \beta, \gamma)-1) f(t)=(1-F(t)) \frac{\kappa}{\kappa-1} Q(t \mid \alpha, \beta, \gamma)-f(t)\left\{\frac{\kappa}{\kappa-1} A(t \mid \alpha, \beta, \gamma)-1\right\} .
$$

After simplification and integration we arrive at

$$
F(t)=1-[A(t \mid \alpha, \beta, \gamma)]^{-\kappa} \quad \text { for } t \geq 0 .
$$

\subsection{Characterizations Based on Truncated Moment of a Function of the Random Variable}

In this subsection, we first present a characterization of the EQHR distribution in terms of a simple relationship between truncated moment of a function of $X$ and another function. This characterization result employs a version of the theorem due to Glänzel [10]; see Theorem A.1 of Appendix A. Note that the result holds also when the interval $H$ is not closed. Moreover, it could be also applied when the cdf $F$ does not have a closed form. As shown by Glänzel [11], this characterization is stable in the sense of weak convergence.

Proposition 6.2. Let $X: \Omega \rightarrow(0, \infty)$ be a continuous random variable and let $q(x)=[A(x \mid \alpha, \beta, \gamma)]^{-1}$ for $x>0$. The random variable $X$ has $p d f(2.3)$ if and only if the function $\eta$ defined in Theorem A.1 has the form

$$
\eta(x)=\frac{\kappa}{\kappa+1}[A(x \mid \alpha, \beta, \gamma)]^{-1}, \quad x>0 .
$$

Proof. Let the random variable $X$ have pdf (2.3), then

$$
(1-F(x)) E(q(X) \mid X \geq x)=\frac{\kappa}{\kappa+1}[A(x \mid \alpha, \beta, \gamma)]^{-(\kappa+1)}, \quad x>0,
$$

or

$$
E(q(X) \mid X \geq x)=\frac{\kappa}{\kappa+1}[A(x \mid \alpha, \beta, \gamma)]^{-1}, \quad x>0,
$$

and

$$
\eta(x)-q(x)=-\frac{1}{\kappa+1}[A(x \mid \alpha, \beta, \gamma)]^{-1}<0 \quad \text { for } x>0 .
$$

Conversely if $\eta$ is given as above, then

$$
\eta^{\prime}(x)=-\frac{\kappa}{\kappa+1} Q(x \mid \alpha, \beta, \gamma)[A(x \mid \alpha, \beta, \gamma)]^{-2}<0 \quad \text { for } x>0,
$$

and

and hence

$$
s^{\prime}(x)=\frac{\eta^{\prime}(x)}{\eta(x)-q(x)}=\frac{-\frac{\kappa}{\kappa+1} Q(x \mid \alpha, \beta, \gamma)[A(x \mid \alpha, \beta, \gamma)]^{-2}}{-\frac{1}{\kappa+1}[A(x \mid \alpha, \beta, \gamma)]^{-1}}=\frac{\kappa Q(x \mid \alpha, \beta, \gamma)}{[A(x \mid \alpha, \beta, \gamma)]}, \quad x>0,
$$

and

$$
s(x)=\ln \left\{[A(x \mid \alpha, \beta, \gamma)]^{\kappa}\right\}, \quad x>0,
$$

$$
e^{-s(x)}=[A(x \mid \alpha, \beta, \gamma)]^{-\kappa}, \quad x>0 .
$$

In view of Theorem A.1, $X$ has density (2.3).

Corollary 6.3. Let $X: \Omega \rightarrow(0, \infty)$ be a continuous random variable. The pdf of $X$ is $(2.3)$ if and only if there exist functions $\eta(x)$ and $q(x)$ defined in Theorem A.1 satisfying the differential equation

$$
\frac{\eta^{\prime}(x)}{\eta(x)-q(x)}=\frac{\kappa Q(x \mid \alpha, \beta, \gamma)}{[A(x \mid \alpha, \beta, \gamma)]}, \quad x>0 .
$$

Remark 6.4. The general solution of the differential equation in Corollary 6.3 is

$$
\eta(x)=[A(x \mid \alpha, \beta, \gamma)]^{\kappa}\left[-\int \frac{\kappa Q(x \mid \alpha, \beta, \gamma)}{[A(x \mid \alpha, \beta, \gamma)]^{\kappa+1}} q(x) d x+D\right],
$$

where $D$ is a constant. Note that a set of functions satisfying the above equation is given in Proposition 6.2 with $D=0$. However, it should also be noted that there are other pairs of functions $(\eta, q)$ satisfying the conditions of Theorem A.1. 


\subsection{Characterization Based on Hazard Function}

It is well known that the hazard function $h_{F}$ of a twice differential distribution function $F$ satisfies the firstorder differential equation $\frac{d}{d x}[\log f(x)]=h_{F}^{\prime}(x) / h_{F}(x)-h_{F}(x)$.

For many univariate continuous distributions, this is the only differential equation available in terms of the hazard function. The following proposition establishes a characterization of the EQHR distribution which is not of the above trivial form.

Proposition 6.5. Let $X: \Omega \rightarrow(0, \infty)$ be a continuous random variable. The pdf of $X$ is $(2.3)$ if and only if its hazard function $h_{F}$ satisfies the first-order differential equation

$$
h_{F}^{\prime}(x)[A(x \mid \alpha, \beta, \gamma)]+h_{F}(x) Q(x \mid \alpha, \beta, \gamma)=\kappa(\beta+2 \gamma x) .
$$

Proof. If $X$ has pdf (2.3), then the above equation holds. Now if the differential equation holds, then

$$
\frac{d}{d x}\left\{h_{F}(x)[A(x \mid \alpha, \beta, \gamma)]\right\}=\kappa \frac{d}{d x} Q(x \mid \alpha, \beta, \gamma),
$$

or

$$
h_{F}(x)=\kappa Q(x \mid \alpha, \beta, \gamma)[A(x \mid \alpha, \beta, \gamma)]^{-1}, \quad x>0,
$$

which is the hazard function of the EQHR distribution.

\subsection{Characterization Based on Mills Ratio}

We introduce a characterization of EQHR via the Mills ratio.

Definition 6.6. Let $X: \Omega \rightarrow(0, \infty)$ be a continuous random variable having absolutely continuous cdf $F(x)$ and pdf $f(x)$. The Mills ratio $m(x)$ of a twice differentiable function $F$ satisfies the first-order differential equation

$$
\frac{d}{d x}[\ln f(x)]=-\left[\frac{1}{m(x)}+\frac{m^{\prime}(x)}{m(x)}\right]
$$

Proposition 6.7. Let $X: \Omega \rightarrow(0, \infty)$ be a continuous random variable. The pdf of $X$ is $(2.3)$ if and only if the Mills ratio satisfies the first-order differential equation

$$
m^{\prime}(x) Q(x \mid \alpha, \beta, \gamma)+m(x)(\beta+\gamma x)=\kappa^{-1} Q(x \mid \alpha, \beta, \gamma) \text {. }
$$

Proof. If $X$ has pdf (2.3), then the above differential equation holds. Now if the differential equation holds, then

$$
\frac{d}{d x}[m(x) Q(x \mid \alpha, \beta, \gamma)]=\kappa^{-1} \frac{d}{d x}[A(x \mid \alpha, \beta, \gamma)],
$$

or

$$
m(x)=\kappa^{-1}[Q(x \mid \alpha, \beta, \gamma)]^{-1}[A(x \mid \alpha, \beta, \gamma)],
$$

which is the Mills ratio of the EQHR distribution.

\subsection{Characterization Based on Certain Functions of the Random Variable}

The following proposition already appeared in [12]. So we will just state it here which can be used to characterize the EQHR distribution.

Proposition 6.8. Let $X: \Omega \rightarrow(0, \infty)$ be a continuous random variable with $c d f(2.2)$. Let $\psi(x)$ be a differentiable function on $(0, \infty)$ with $\lim _{x \rightarrow 0^{+}} \psi(x)=1$. Then for $\delta \neq 0, E(\psi(X) \mid X>x)=\delta \psi(x), x>0$, if and only if $\psi(x)=(1-F(x))^{1 / \delta-1}, x>0$. 
Remark 6.9. It is easy to see that for certain functions, e.g. $\psi(x)=[A(x \mid \alpha, \beta, \gamma)]^{-1}$ and $\delta=\frac{\kappa}{1+\kappa}$, Proposition 6.8 provides a characterization of the EQHR distribution. Clearly there are other functions $\psi(x)$, we chose the above one for simplicity.

Proposition 6.10. Let $X: \Omega \rightarrow(0, \infty)$ be a continuous random variable with $c d f F$ and $p d f f$. Let $\psi(x)$ and $\varphi(x)$ be differentiable functions on $(0, \infty)$ such that

$$
\int_{0}^{\infty} \frac{\varphi^{\prime}(x)}{\varphi(x)-\psi(x)} d x=\infty
$$

Then $E(\psi(X) \mid X>x)=\varphi(x), x>0$ implies

$$
F(x)=1-\exp \left[-\int_{0}^{x} \frac{\varphi^{\prime}(t)}{\varphi(t)-\psi(t)} d t\right], \quad x \geq 0 .
$$

Proof. We have

$$
\int_{x}^{\infty} \psi(u) f(u) d u=(1-F(x)) \varphi(x) .
$$

Differentiating both sides of the above equation with respect to $x$ and then rearranging the terms, we arrive at

$$
\frac{f(x)}{1-F(x)}=\frac{\varphi^{\prime}(x)}{\varphi(x)-\psi(x)}, \quad x>0 .
$$

Integrating the last equation from 0 to $x$, we have

$$
F(x)=1-\exp \left[-\int_{0}^{x} \frac{\varphi^{\prime}(t)}{\varphi(t)-\psi(t)} d t\right], \quad x \geq 0 .
$$

Remark 6.11. Taking $\psi(x)=[A(x \mid \alpha, \beta, \gamma)]^{\kappa / 2}$ and $\varphi(x)=2 \psi(x)$, we see that Proposition 6.10 provides a characterization of (2.2). Clearly there are other choices of these functions.

\subsection{Characterization Through Distribution of the First-Order Statistic}

Here we characterize the EQHR distribution via a distribution of first-order statistic.

Proposition 6.12. Let $X_{1}, X_{2}, \ldots, X_{n}$ be i.i.d. random variables. If the distribution of the $X_{i}$ is $E Q H R$, then $X_{1: n}$ has EQHR distribution.

Proof. Observe that

$$
\begin{aligned}
& P_{r}\left(X_{1: n}>x\right)=\left[P_{r}\left(X_{1}>x\right)\right]^{n}=[1-F(x)]^{n}, \\
& P_{r}\left(X_{1: n}>x\right)=[A(x \mid \alpha, \beta, \gamma)]^{-n \kappa}, \quad x>0 .
\end{aligned}
$$

\subsection{Characterization via Conditional Expectation of Record Values}

Nagaraja [18], Arnold, Balakrishnan, and Nagarajah [3], Khan and Alzaid [14], and Athar and Noor [4] characterized distributions via conditional expectation of record values.

Proposition 6.13. Let $X: \Omega \rightarrow(0, \infty)$ be a continuous random variable with $c d f F(x)$ and $p d f f(x)$. Let $X_{U(r)}$ be the rth record value of a random sample $X_{1}, X_{2}, \ldots, X_{n}$. Then for two successive values $X_{U(r)}$ and $X_{U(s)}$, $1 \leq r<s \leq n$, one has

$$
E\left[\left(h\left(X_{U(s)}-X_{U(r)}\right)\right)^{q} \mid X_{U(r)}=x\right]=a^{*} \sum_{j=0}^{q}\left(\begin{array}{l}
q \\
j
\end{array}\right)(h(x))^{q-j}\left(\frac{b}{a}\right)^{j}
$$

if and only if $F(x)=1-[a+b h(x)]^{c}, a \neq 0$, where $a^{*}=\sum_{i=0}^{q}\left(\begin{array}{c}q \\ i\end{array}\right)(-1)^{i+q}\left(\frac{c}{c+i}\right)^{s-r}$ and $h(x)$ is a differentiable function of $x$. 
Remark 6.14. Taking $a=1, b=1, h(x)=[A(x \mid \alpha, \beta, \gamma)-1], c=-\kappa$, we see that Proposition 6.13 provides a characterization of $F(x)=1-[A(x \mid \alpha, \beta, \gamma)]^{-\kappa}, x \geq 0$.

\section{Compounding}

Here we derive the EQHR distribution through compounding (i) GQHR and gamma distributions and (ii) GQHR and sized biased moment Erlang distributions.

Theorem 7.1. Let $w(x \mid \alpha, \beta, \gamma, \theta)=\theta Q(x \mid \alpha, \beta, \gamma) e^{-\theta[A(x \mid \alpha, \beta, \gamma)-1]}, x>0$, be pdf of GQHR distribution and let $\theta$ have the gamma distribution with $p d f$

$$
g(\theta / \kappa)=\frac{1}{\Gamma(\kappa)} \theta^{\kappa-1} e^{-\theta}, \quad \theta>0 .
$$

Then X has pdf (2.3).

Proof. For the compounding pdf, we have

$$
\begin{aligned}
& f(x, \alpha, \beta, \gamma, \kappa)=\int_{0}^{\infty} w(x \mid \alpha, \beta, \gamma, \theta) g(\theta / \kappa) d \theta, \\
& f(x, \alpha, \beta, \gamma, \kappa)=\int_{0}^{\infty} \theta Q(x \mid \alpha, \beta, \gamma) e^{-\theta[A(x \mid \alpha, \beta, \gamma)-1]} \frac{1}{\Gamma(\kappa)} \theta^{\kappa-1} e^{-\theta} d \theta, \\
& f(x, \alpha, \beta, \gamma, \kappa)=\kappa Q(x \mid \alpha, \beta, \gamma)[A(x \mid \alpha, \beta, \gamma)]^{-(\kappa+1)}, \quad x>0,
\end{aligned}
$$

which is the pdf of the EQHR distribution.

Theorem 7.2. Let $w(x \mid \alpha, \beta, \gamma, \theta)=\theta Q(x \mid \alpha, \beta, \gamma) e^{-\theta[A(x \mid \alpha, \beta, \gamma)-1]}, x>0$, be $p d f$ of GQHR distribution and let $\theta$ have sized biased moment Erlang distribution with $p d f$

$$
g(\theta / \tau, \kappa)=\frac{1}{\Gamma(\kappa-\tau)} \theta^{\kappa-\tau-1} e^{-\theta}, \quad \theta>0 .
$$

Then $X$ has pdf (2.3).

Proof. For the compounding pdf, we have

$$
\begin{aligned}
& f(x, \alpha, \beta, \gamma, \tau, \kappa)=\int_{0}^{\infty} w(x \mid \alpha, \beta, \gamma, \theta) g(\theta / \tau, \kappa) d \theta, \\
& f(x, \alpha, \beta, \gamma, \tau, \kappa)=\int_{0}^{\infty} \theta Q(x \mid \alpha, \beta, \gamma) e^{-\theta[A(x \mid \alpha, \beta, \gamma)-1]} \frac{1}{\Gamma(\kappa-\tau)} \theta^{\kappa-\tau-1} e^{-\theta} d \theta, \\
& f(x, \alpha, \beta, \gamma, \tau, \kappa)=(\kappa-\tau) Q(x \mid \alpha, \beta, \gamma)[A(x \mid \alpha, \beta, \gamma)]^{-(\kappa-\tau+1)}, \quad x>0 .
\end{aligned}
$$

For $\tau=0$, we have

$$
f(x, \alpha, \beta, \gamma, \kappa)=\kappa Q(x \mid \alpha, \beta, \gamma)[A(x \mid \alpha, \beta, \gamma)]^{-(\kappa+1)}, \quad x>0,
$$

which is the pdf of the EQHR distribution.

\section{Simulation Study}

We generate 1000 random numbers from the EQHR distribution $(\alpha=0.01, \beta=0.5, \gamma=2, \kappa=5)$ with the R-Package. The MLEs of $\alpha, \beta, \gamma, \kappa$ using the 1000 random numbers generated from the EQHR distribution are

$$
\hat{\alpha}=0.008365965, \quad \hat{\beta}=0.516160379, \quad \hat{\gamma}=1.896250595, \quad \hat{\kappa}=5.275523408,
$$


and the standard errors of the MLEs are

$$
\operatorname{se}(\hat{\alpha})=0.01016965, \quad \operatorname{se}(\hat{\beta})=0.14180229, \quad \operatorname{se}(\hat{\gamma})=0.61733376, \quad \operatorname{se}(\hat{\kappa})=1.00287433 .
$$

\section{Maximum Likelihood Estimation}

In this section, parameter estimates are derived using the maximum likelihood method. The log-likelihood function for the vector of parameters $\Phi=(\alpha, \beta, \gamma, \kappa)$ of EQHR is

$$
\ln \mathrm{L}(\Phi)=n \ln (\kappa)+\sum_{i=1}^{n} \ln Q\left(x_{i} \mid \alpha, \beta, \gamma\right)-(\kappa+1) \sum_{i=1}^{n} \ln \left[A\left(x_{i} \mid \alpha, \beta, \gamma\right)\right]
$$

In order to compute the estimates of parameters of the EQHR distribution, the following nonlinear equations must be solved simultaneously:

$$
\begin{aligned}
\frac{\partial}{\partial \alpha}(\operatorname{lnL}(\Phi)) & =\sum_{i=1}^{n}\left[Q\left(x_{i} \mid \alpha, \beta, \gamma\right)\right]^{-1}-(\kappa+1) \sum_{i=1}^{n} x_{i}\left[A\left(x_{i} \mid \alpha, \beta, \gamma\right)\right]^{-1}=0, \\
\frac{\partial}{\partial \beta}(\operatorname{lnL}(\Phi)) & =\sum_{i=1}^{n} x_{i}\left[Q\left(x_{i} \mid \alpha, \beta, \gamma\right)\right]^{-1}-(\kappa+1) \sum_{i=1}^{n} \frac{x_{i}^{2}}{2}\left[A\left(x_{i} \mid \alpha, \beta, \gamma\right)\right]^{-1}=0, \\
\frac{\partial}{\partial \gamma}(\operatorname{lnL}(\Phi)) & =\sum_{i=1}^{n} x_{i}^{2}\left[Q\left(x_{i} \mid \alpha, \beta, \gamma\right)\right]^{-1}-(\kappa+1) \sum_{i=1}^{n} \frac{x_{i}^{3}}{3}\left[A\left(x_{i} \mid \alpha, \beta, \gamma\right)\right]^{-1}=0, \\
\frac{\partial}{\partial \kappa}(\operatorname{lnL}(\Phi)) & =\frac{n}{\kappa}-\sum_{i=1}^{n} \ln \left[A\left(x_{i} \mid \alpha, \beta, \gamma\right)\right]=0 .
\end{aligned}
$$

\subsection{Failure Times of 84 Aircraft Windshield}

The EQHR distribution is compared with sub-models. Different goodness fit measures like Cramer-von Mises (W), Anderson Darling (A), Kolmogorov-Smirnov (K-S) statistics with p-values, Akaike information criterion (AIC), consistent Akaike information criterion (CAIC), Bayesian information criterion (BIC), Hannan-Quinn information criterion (HQIC) and likelihood ratio statistics are computed for failure times of 84 Aircraft Windshield using R-Package. Tahir et al. [22] and Abouelmagd et al. [1] also used failure times of 84 Aircraft Windshield in their papers. The data values are:

$0.040,1.866,2.385,3.443,0.301,1.876,2.481,3.467,0.309,1.899,2.610,3.478,0.557,1.911$,

$2.625,3.578,0.943,1.912,2.632,3.595,1.070,1.914,2.646,3.699,1.124,1.981,2.661,3.779$,

$1.248,2.010,2.688,3.924,1.281,2.038,2.82,3,4.035,1.281,2.085,2.890,4.121,1.303,2.089$,

2.902, 4.167, 1.432, 2.097, 2.934, 4.240, 1.480, 2.135, 2.962, 4.255, 1.505, 2.154, 2.964, 4.278,

1.506, 2.190, 3.000, 4.305, 1.568, 2.194, 3.103, 4.376, 1.615, 2.223, 3.114, 4.449, 1.619, 2.224,

3.117, 4.485, 1.652, 2.229, 3.166, 4.570, 1.652, 2.300, 3.344, 4.602, 1.757, 2.324, 3.376, 4.663.

The better fit corresponds to smaller W, A, K-S, AIC, CAIC, BIC, HQIC and $-\ell$ values. The maximum likelihood estimates (MLEs) of unknown parameters and values of goodness of fit measures are computed for EQHR and sub-models. The MLEs, their standard errors (in parentheses) and goodness-of-fit statistics are given in Table 2. Table 3 displays goodness-of-fit values.

The EQHR distribution is better fitted than sub-models because the values of all criteria of goodness of fit (except W) are significantly smaller for the EQHR distribution. 


\begin{tabular}{|c|c|c|c|c|c|c|c|c|}
\hline Model & $\alpha$ & $\beta$ & $\gamma$ & $\kappa$ & $\mathbf{w}$ & A & K-S & P-value \\
\hline QHR & $\begin{array}{l}0.004022088 \\
(0.003414835)\end{array}$ & $\begin{array}{l}1.000000 \mathrm{e}-10 \\
(0.008027466)\end{array}$ & $\begin{array}{l}0.009061885 \\
(0.005700021)\end{array}$ & $\begin{array}{l}11.97585 \\
(6.875017327)\end{array}$ & 0.06068681 & 0.4635632 & 0.0786 & 0.6694 \\
\hline QHR & $\begin{array}{l}0.0301076294 \\
(0.03076228)\end{array}$ & $\begin{array}{l}0.0000000001 \\
(0.12762154)\end{array}$ & $\begin{array}{l}0.1998662852 \\
(0.06157796)\end{array}$ & 1 & 0.105176 & 0.9236758 & 0.1267 & 0.1308 \\
\hline Lomax & 1 & 0 & 0 & $\begin{array}{l}0.8225503 \\
(0.08921798)\end{array}$ & 0.5765111 & 3.867886 & 0.404 & $1.771 \mathrm{e}-12$ \\
\hline E-Exp & $\begin{array}{l}0.006914977 \\
(0.003569097)\end{array}$ & 0 & 0 & $\begin{array}{l}57.022086278 \\
(29.777706756)\end{array}$ & 0.1712903 & 1.429851 & 0.305 & $2.707 e-07$ \\
\hline E-Ray & 0 & $\begin{array}{l}0.0076178 \\
(0.003801804)\end{array}$ & 0 & $\begin{array}{l}34.2765810 \\
(17.039219026)\end{array}$ & 0.07188289 & 0.7165626 & 0.1163 & 0.2002 \\
\hline$E-W$ & 0 & 0 & $\begin{array}{l}0.02004073 \\
(0.02063317)\end{array}$ & $\begin{array}{l}6.62888946 \\
(5.97855420)\end{array}$ & 0.05610142 & 0.5784477 & 0.0936 & 0.4453 \\
\hline LFR & $\begin{array}{l}0.0007443581 \\
(0.00299964)\end{array}$ & $\begin{array}{l}0.0646225644 \\
(0.01998114)\end{array}$ & 0 & $\begin{array}{l}4.2662076477 \\
(1.12073841)\end{array}$ & 0.100075 & 0.9389629 & 0.1218 & 0.1608 \\
\hline
\end{tabular}

Table 2: MLEs, their standard errors (in parentheses) and goodness-of-fit statistics for data set I.

\begin{tabular}{llllll}
\hline Model & AIC & CAIC & BIC & HQIC & $-\ell$ \\
\hline EQHR & 264.1326 & 264.6326 & 273.9032 & 268.0626 & 128.0663 \\
QHR & 280.369 & 280.6653 & 287.697 & 283.3165 & 137.1845 \\
Lomax & 411.8832 & 411.9314 & 414.3259 & 412.8657 & 204.9416 \\
E-Exp & 335.1877 & 335.3341 & 340.073 & 337.1527 & 165.5939 \\
E-Ray & 271.4702 & 271.6166 & 276.3555 & 273.4352 & 133.7351 \\
E-W & 273.2853 & 273.4316 & 278.1706 & 275.2503 & 134.6426 \\
LFR & 281.1604 & 281.4567 & 288.4884 & 284.1079 & 137.5802 \\
\hline
\end{tabular}

Table 3: Goodness-of-fit statistics for data set I.

\subsection{Failure Times of Components}

The failure times (Murthy, Xie and Jiang [16]) of 50 components (per $1000 \mathrm{~h}$ ) are:

0.036, 0.058, 0.061, 0.074, 0.078, 0.086, 0.102, 0.103, 0.114, 0.116, 0.148, 0.183, 0.192, 0.254,

$0.262,0.379,0.381,0.538,0.570,0.574,0.590,0.618,0.645,0.961,1.228,1.600,2.006,2.054$,

2.804, 3.058, 3.076, 3.147, 3.625, 3.704, 3.931, 4.073, 4.393, 4.534, 4.893, 6.274, 6.816, 7.896,

7.904, 8.022, 9.337, 10.940, 11.020, 13.880, 14.730, 15.080.

The better fit corresponds to smaller W, A, K-S, AIC, CAIC, BIC, HQIC and $-\ell$ values. The maximum likelihood estimates (MLEs) of unknown parameters and values of goodness of fit measures are computed for EQHR and sub-models. The MLEs, their standard errors (in parentheses) and goodness-of-fit statistics are given in Table 4. Table 5 displays goodness-of-fit values.

The EQHR distribution is better fitted than sub-models because the values of all criteria of goodness of fit (except BIC) are significantly smaller for the EQHR distribution.

\section{Concluding Remarks}

We proposed an EQHR distribution with bathtub hazard rate function. We studied certain structural properties, stochastic ordering, descriptive measures, reliability measures, compounding and characterizations of the EQHR distribution. A simulation study was carried out. Maximum likelihood estimates were computed. Goodness of fit shows that the EQHR distribution is a better fit. Applications of the EQHR model to failure times were illustrated to show significance and flexibility of the EQHR distribution. 


\begin{tabular}{|c|c|c|c|c|c|c|c|c|}
\hline Model & $\alpha$ & $\beta$ & $\gamma$ & $\kappa$ & W & A & K-S & P-value \\
\hline EQHR & $\begin{array}{l}2.7447282511 \\
(0.77354560)\end{array}$ & 0.0000000001 & $\begin{array}{l}0.2614325281 \\
(0.22026842)\end{array}$ & $\begin{array}{l}0.4888605886 \\
(0.03419087)\end{array}$ & 0.1368555 & 0.8672208 & 0.1249 & 0.3844 \\
\hline QHR & $\begin{array}{l}0.7985513974 \\
(0.22248409)\end{array}$ & $\begin{array}{l}0.0000000001 \\
(0.29856803)\end{array}$ & $\begin{array}{l}0.0167545084 \\
(0.02284814)\end{array}$ & 1 & 0.1579334 & 0.9786611 & 0.1426 & 0.2377 \\
\hline Lomax & 1 & 0 & 0 & $\begin{array}{l}0.9436866 \\
(0.1334573)\end{array}$ & 0.2217343 & 1.378065 & 0.1566 & 0.1547 \\
\hline E-Exp & $\begin{array}{l}0.7693872 \\
(0.5962221)\end{array}$ & 0 & 0 & $\begin{array}{l}1.0770320 \\
(0.4588102)\end{array}$ & 0.2158481 & 1.339918 & 0.1501 & 0.1895 \\
\hline E-Ray & 0 & $\begin{array}{l}124.2841497 \\
(77.72826236)\end{array}$ & 0 & $\begin{array}{l}0.2194352 \\
(0.03965866)\end{array}$ & 0.331417 & 2.003366 & 0.1831 & 0.06124 \\
\hline E-W & 0 & 0 & $\begin{array}{l}124.2841497 \\
(3552.3752707)\end{array}$ & $\begin{array}{l}0.2194352 \\
(0.0206158)\end{array}$ & 0.3617802 & 2.13793 & 0.1849 & 0.0572 \\
\hline LFR & $\begin{array}{l}1.6892798 \\
(1.2345675)\end{array}$ & $\begin{array}{l}0.3461011 \\
(0.5747975)\end{array}$ & & $\begin{array}{l}0.6335410 \\
(0.2512073)\end{array}$ & 0.2014193 & 1.252045 & 0.1511 & 0.1841 \\
\hline
\end{tabular}

Table 4: MLEs, their standard errors (in parentheses) and goodness-of-fit statistics for data set II.

\begin{tabular}{llllll}
\hline Model & AIC & CAIC & BIC & HQIC & $-\ell$ \\
\hline EQHR & 210.7647 & 211.6536 & 218.4128 & 213.6771 & 101.3823 \\
QHR & 212.1595 & 212.6813 & 217.8956 & 214.3439 & 103.0798 \\
Lomax & 213.7636 & 213.8469 & 215.6756 & 214.4917 & 105.8818 \\
E-Exp & 215.6431 & 215.8984 & 219.4672 & 217.0993 & 105.8216 \\
E-Ray & 223.3178 & 223.5732 & 227.1419 & 224.7741 & 109.6589 \\
E-W & 224.641 & 224.8963 & 228.465 & 226.0972 & 110.3205 \\
LFR & 215.0545 & 215.5763 & 220.7906 & 217.2389 & 104.5273 \\
\hline
\end{tabular}

Table 5: Goodness-of-fit statistics for data set II.

\section{A Appendix}

Theorem A.1. Let $(\Omega, F, P)$ be a given probability space and let $H=[a, b]$ be an interval for some $a<b$ $(a=-\infty, b=\infty$ might as well be allowed). Let $X: \Omega \rightarrow H$ be a continuous random variable with distribution function $F$. Let $q$ be a real function defined on $H$ such that $E[q(X) \mid X \geq x]=\eta(x), x \in H$, is defined with some real function $\eta$. Assume that $q \in C^{1}(H), \eta \in C^{2}(H)$ and $F$ is a twice continuously differentiable and strictly monotone function on the set $H$. Finally, assume that the equation $\eta=q$ has no real solution in the interior of $H$. Then $F$ is uniquely determined by the functions $q$ and $\eta$, particularly

$$
F(x)=\int_{a}^{x} C\left|\frac{\eta^{\prime}(u)}{\eta(u)-q(u)}\right| \exp (-s(u)) d u,
$$

where the function $s$ is a solution of the differential equation $s^{\prime}=\frac{\eta^{\prime}}{\eta-q}$ and $C$ is the normalization constant, such that $\int_{H} d F=1$.

\section{References}

[1] T. H. M. Abouelmagd, S. Al-Mualim, M. Elgarhy, A. Z. Afify and M. Ahmad, Properties of the four-parameter Weibull distribution and its applications, Pakistan J. Statist. 33 (2017), 449-466.

[2] A. Al-Hossain and J. G. Dar, Some results on moment of order statistics for the quadratic hazard rate distribution, J. Stat. Appl. 5 (2016), 371-376.

[3] B. C. Arnold, N. Balakrishnan and H. Nagarajah, Records, John Wiley, New York, 1998.

[4] H. Athar and Z. Noor, Characterization of probability distributions by conditional expectations of functions of record statistics, J. Egyptian Math. Soc. 22 (2014), 275-279. 
[5] L. J. Bain, Analysis for the linear failure-rate life-testing distribution, Technometrics 16 (1974), 551-559.

[6] G. K. Bhattacharyya and R. A. Johnson, Estimation of reliability in a multicomponent stress-strength model, J. Amer. Statist. Assoc. 69 (1974), 966-970.

[7] F. A. Bhatti and M. Ahmad, Some characterizations of quadratic hazard rate-geometric (Qhr-G) distribution, Int. J. Eng. Res. Gen. Sci. 5 (2017), 164-171.

[8] M. A. El-Damcese and D. A. Ramadan, Reliability analysis using the generalized quadratic hazard rate truncated Poisson maximum distribution, Int. J. Stat. Appl. 5 (2015), 302-316.

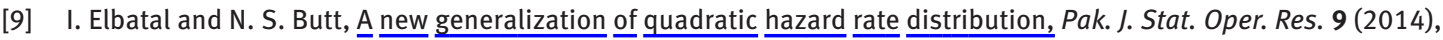
343-361.

[10] W. Glänzel, A characterization theorem based on truncated moments and its application to some distribution families, in: Mathematical Statistics and Probability Theory, D. Reidel, Dordrecht (1987), 75-84.

[11] W. Glänzel, Some consequences of a characterization theorem based on truncated moments, Statistics 21 (1990), 613-618.

[12] G. G. Hamedani, On certain generalized gamma convolution distributions II, Technical Report 484, Marquette University, 2013.

[13] M. Kayid, I. Elbatal and F. Merovci, A new family of generalized quadratic hazard rate distribution with applications, J. Testing Eval. 44 (2016), 1733-1744.

[14] A. H. Khan and A. A. Alzaid, Characterization of distributions through linear regression of non-adjacent generalized order statistics, J. Appl. Statist. Sci. 13 (2004), 123-136.

[15] F. Merovci and I. Elbatal, The beta quadratic hazard rate distribution, Pakistan J. Statist. 31 (2015), 427-446.

[16] D. Murthy, M. Xie and R. Jiang, Weibull Models, Wiley Ser. Probab. Stat., John Wiley \& Sons, New York, 2004.

[17] A. Mustafa, A new bivariate distribution with generalized quadratic hazard rate marginals distributions, Journal of Math. Stat. 12 (2016), 255-270.

[18] H. Nagarajah, Some characterizations of continuous distributions based on regressions of adjacent order statistics and record values, Sankhya A 50 (1988), 70-73.

[19] H. M. Okasha, M. Kayid, M. A. Abouammoh and I. Elbatal, A new family of quadratic hazard rate-geometric distributions with reliability applications, J. Testing Eval. 44 (2015), 1937-1948.

[20] R. Roozegar and S. Nadarajah, The quadratic hazard rate power series distribution, J. Testing Eval. 45 (2016), $1058-1072$.

[21] A. M. Sarhan, Generalized quadratic hazard rate distribution, Int. J. Appl. Math. Stat. 14 (2009), 94-106.

[22] M. H. Tahir, G. M. Cordeiro, M. Mansoor and M. Zubair, The Weibull-Lomax distribution: Properties and applications, Hacet. J. Math. Stat. 44 (2015), 461-480. 